\title{
Histoscope: A Web-Based Microscopy Tool for Oral Histology Education
}

\author{
Nazlee Sharmin, Ava K. Chow, Alice S. Dong, Nadine C. Milos \\ School of Dentistry, Faculty of Medicine and Dentistry, University of Alberta, Edmonton, Canada
}

Objectives: Histology, the study of tissue structure under a microscope, is one of the most essential yet least engaging topics for health professional students. Understanding tissue microanatomy is crucial for students to be able to recognize cellular structures and follow disease pathogenesis. Traditional histology teaching labs rely on light microscopes and a limited array of slides, which inhibits simultaneous observation by multiple learners, and prevents in-class discussions. We have developed an interactive web-based microscopy tool called "Histoscope" for oral histology in this context. Methods: Good quality microscope slides were selected for digital scanning. The slides were scanned with multiple layers of $z$-stacking, a method of taking multiple images at different focal distances. The digital images were checked for quality and were archived on Histoscope. The slides were annotated, and self-assessment questions were prepared for the website. Interactive components were programmed on the website to mimic the experience of using a real light microscope. Results: This web-based tool allows users to interact with histology slides, replicating the experience of observing and manipulating a slide under a real microscope. Through this website, learners can access a broad array of digital oral histology slides and self-assessment questions. Conclusions: Incorporation of Histoscope in a course can shift traditional teacher-centered histology learning to a collaborative and student-centered learning environment. This platform can also provide students the flexibility to study histology at their own pace.

Keywords: Histology, Education, Web-Based Microscope, Self-Assessment, Dental Education

Submitted: December 22, 2020

Revised: February 10, 2021

Accepted: March 2, 2021

\section{Corresponding Author}

Nazlee Sharmin

School of Dentistry, Faculty of Medicine and Dentistry, University of Alberta, 5-533, Edmonton Clinic Health Academy (ECHA), 11405 87 Avenue NW, Edmonton, AB T6G 1C9, Canada. Tel: +1-780-4926428,E-mail: nazlee@ualberta.ca (https://orcid.org/0000-00022408-2333)

This is an Open Access article distributed under the terms of the Creative Commons Attribution Non-Commercial License (http://creativecommons.org/licenses/by$\mathrm{nc} / 4.0 /$ ) which permits unrestricted non-commercial use, distribution, and reproduction in any medium, provided the original work is properly cited.

(c) 2021 The Korean Society of Medical Informatics

\section{Introduction}

Histology, the study of tissue structure under a microscope, has always been the principal approach to the structural analysis of living organisms at the cellular level. Understanding tissue microanatomy is essential to train medical, dental, and other health professional students to recognize cellular structures, comprehend the structure-function relationship of cells and tissues, and follow disease pathogenesis. However, this branch of science has been described as one of the least engaging topics by health professional students [1]. The traditional method of teaching histology relies on light microscopes, which does not allow simultaneous observation by multiple people, prevents interactive in-class discussions, and thus fails to motivate students [2].

At the School of Dentistry at the University of Alberta, 
students extensively study oral histology to learn about teeth and surrounding oral structures, their development, and developmental anomalies. Besides regular didactic lectures, students attend histology lab sessions in which they share a limited number of light microscopes and glass slides to view tooth and oral structures. The microscopic slides used for teaching are decades old, with some being extremely rare and irreplaceable.

A growing number of universities and medical schools worldwide are embracing digital slides as an alternative to traditional light microscopes $[3,4]$. The incorporation of virtual microscopy in histology and pathology courses has improved in-class teaching environments $[3,5]$ and student performance [6]. The number of websites archiving digital histology slides is also growing internationally. For example, "The SecondLook" is a collection of histology slides developed by the University of Michigan [7]. "Oral Histology" created by the University of Kentucky College of Medicine contains a collection of annotated but low-resolution images of oral histology slides [8]. However, the existing web-based histology databases lack sufficient, high-quality resources from the oral cavity, which are critical for dentistry students. Most of the publicly available histology databases provide either annotated or non-annotated images without the ability to switch from one to the other, which does not allow learners to perform active memory recall after learning. Other drawbacks of the existing virtual microscopy websites are the quality of graphical resolution [9] and lack of z-axis information, which is essential for reconstructing the three-dimensional visualization of cellular structure [10]. Moreover, in most online sources, users cannot manipulate images to observe structural details. The few websites that provide the ability to zoom in and out permit users to expand a picture only at a predefined location. The inability to magnify any region of interest limits learners' freedom to explore the histological section and restricts their curiosity, reducing the student-centeredness of their learning. Furthermore, the COVID-19 pandemic has moved most university teaching online, necessitating an alternative way to teach histology virtually yet in an interactive and engaging manner.

In this context, we have developed a web-based interactive microscopy tool called "Histoscope." We have scanned our existing oral histological slides in high resolution and archived them on our website. The site allows users to interact with the digital slides and self-assess their knowledge of oral histology.

\section{Methods}

\section{Slide Digitalization and Organization}

The School of Dentistry has a collection of oral histology glass slides to teach histology labs with light microscopes. We curated those slides for quality and rarity and selected the best slides for whole-slide scanning using an Aperio Digital Slide Scanner (Leica Biosystems, Wetzlar, Germany). We chose the slides to represent the oral mucosa, oral structures, tooth, tooth development, and facial structure development. The slides were scanned with 25 layers of $z$-stacking, an image-processing method of taking multiple images at different focal distances and combining them to make a composite image. This technique, also known as focus stacking, is useful for capturing in-focus images of objects under high magnification as the depth of field decreases with magnification [11].

The histology glass slides were scanned under $20 \times$ or $40 \times$ magnification depending on the specimen's size and the

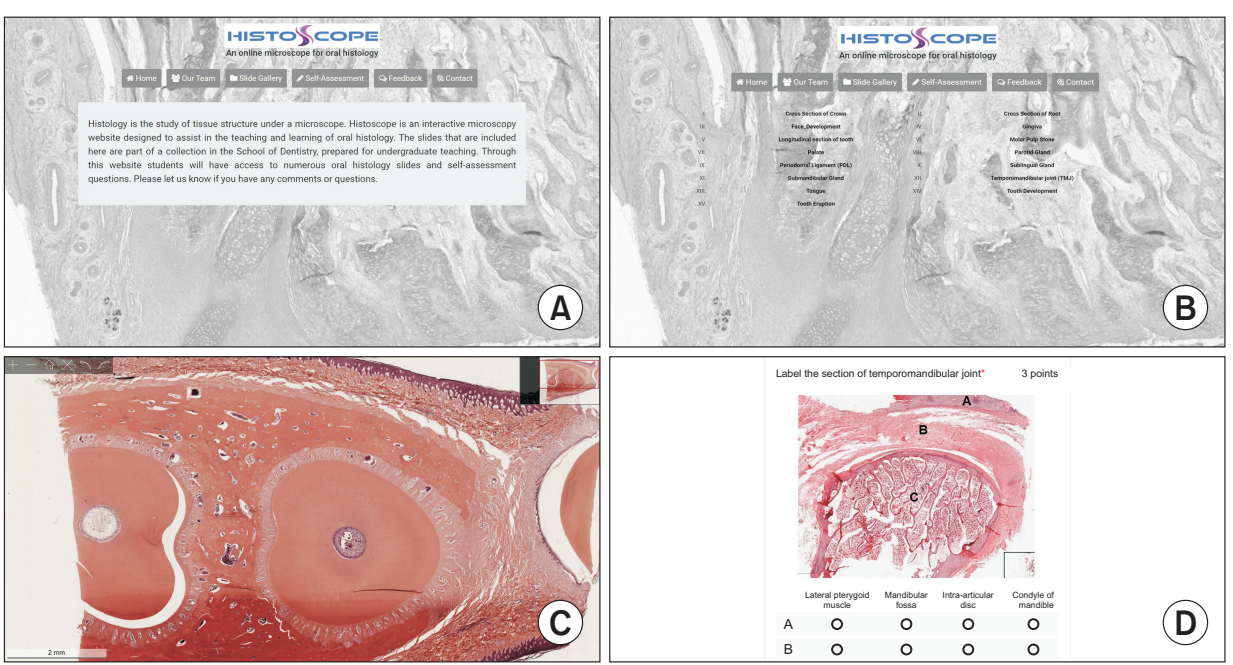

Figure 1. Snapshots from the Histoscope website. (A, B) The contents of the website are organized under six tabs. (B) Digital slides are arranged in 15 groups under the "Slide Gallery" tab. (C) Snapshot of a slide showing the crosssection of a dental root. (D) Sample question from the Self-Assessment section of the website (D). 
structural details required for teaching oral histology. The slides scanned under $40 \times$ magnification has a resolution of 0.247 microns per pixel (MPP); the resolution was 0.497
MPP for the slides scanned under $20 \times$ magnification. The image bit depth was 8 bits for all the digital slides.

Digital scans of the slides were further checked for quality

Table 1. List of digital slides included in the "Slide Gallery" of Histoscope

\begin{tabular}{|c|c|c|c|}
\hline \multirow[b]{2}{*}{ Digital slides } & \multicolumn{3}{|c|}{ Properties } \\
\hline & $\begin{array}{c}\text { Scanning } \\
\text { magnification }\end{array}$ & $\begin{array}{l}\text { Resolution } \\
\text { (MPP) }\end{array}$ & $\begin{array}{c}\text { Pixel } \\
\text { dimension }\end{array}$ \\
\hline \multicolumn{4}{|l|}{ Cross section of crown } \\
\hline Cross section of tooth crown (incisor) & $20 \times$ & 0.497 & $17,987 \times 13,230$ \\
\hline Cross section of a tooth crown (premolar) & $20 \times$ & 0.497 & $35,975 \times 28,613$ \\
\hline Cross section of occlusal surface (molar) & $20 \times$ & 0.497 & $21,585 \times 19,294$ \\
\hline \multicolumn{4}{|l|}{ Cross section of root } \\
\hline Cross section of root I & $40 \times$ & 0.247 & $37,800 \times 21,881$ \\
\hline Cross section of root II & $40 \times$ & 0.247 & $39,600 \times 24,359$ \\
\hline Cross section of root III & $40 \times$ & 0.247 & $50,399 \times 28,112$ \\
\hline Cross section of root, periodontal ligament, alveolar bone I & $40 \times$ & 0.247 & $54,000 \times 49,764$ \\
\hline Cross section of root, periodontal ligament, alveolar bone II & $40 \times$ & 0.247 & $52,199 \times 33,395$ \\
\hline Cross section of root, periodontal ligament, alveolar bone III & $40 \times$ & 0.247 & $50,399 \times 39,356$ \\
\hline \multicolumn{4}{|l|}{ Face development } \\
\hline Cross section of embryo head (day 23) & $20 \times$ & 0.497 & $8,993 \times 9,648$ \\
\hline Cross section of embryo head (developing tongue, palate) I & $20 \times$ & 0.497 & $10,792 \times 13,916$ \\
\hline Cross section of embryo head (developing tongue, palate) II & $20 \times$ & 0.497 & $10,972 \times 13,919$ \\
\hline Cross section of embryo head (developing tongue, palate) III & $20 \times$ & 0.497 & $12,591 \times 15,064$ \\
\hline Cross section of embryo head (developing tongue, palate) IV & $20 \times$ & 0.497 & $10,792 \times 15,729$ \\
\hline Cross section of embryo head (face development) I & $20 \times$ & 0.497 & $21,585 \times 13,619$ \\
\hline Cross section of embryo head (face development) II & $20 \times$ & 0.497 & $21,585 \times 13,045$ \\
\hline \multicolumn{4}{|l|}{ Gingiva } \\
\hline Longitudinal section of tooth, gingiva I & $20 \times$ & 0.497 & $55,762 \times 28,349$ \\
\hline Longitudinal section of tooth, gingiva II & $20 \times$ & 0.497 & $52,164 \times 28,198$ \\
\hline Longitudinal section of tooth, gingiva III & $20 \times$ & 0.497 & $57,561 \times 25,626$ \\
\hline \multicolumn{4}{|l|}{ Longitudinal tooth section } \\
\hline Longitudinal ground section of tooth I & $20 \times$ & 0.497 & $34,176 \times 15,701$ \\
\hline Longitudinal ground section of tooth II & $20 \times$ & 0.497 & $41,372 \times 19,779$ \\
\hline Oblique section of tooth & $20 \times$ & 0.497 & $26,981 \times 20,181$ \\
\hline Longitudinal ground section of tooth III & $20 \times$ & 0.497 & $34,176 \times 18,663$ \\
\hline \multicolumn{4}{|l|}{ Pulp stone } \\
\hline Tooth cross section with pulp stone I & $40 \times$ & 0.247 & $48,600 \times 33,743$ \\
\hline Tooth cross section with pulp stone II & $40 \times$ & 0.247 & $66,599 \times 47,051$ \\
\hline \multicolumn{4}{|l|}{ Palate } \\
\hline Anterior palate I & $40 \times$ & 0.247 & $28,800 \times 78,829$ \\
\hline Anterior palate II & $40 \times$ & 0.247 & $28,800 \times 78,830$ \\
\hline Anterior palate III & $40 \times$ & 0.247 & $23,400 \times 78,854$ \\
\hline Anterior palate IV & $40 \times$ & 0.247 & $23,400 \times 78,856$ \\
\hline
\end{tabular}


Table 1. Continued 1

\begin{tabular}{|c|c|c|c|}
\hline \multirow[b]{2}{*}{ Digital slides } & \multicolumn{3}{|c|}{ Properties } \\
\hline & $\begin{array}{c}\text { Scanning } \\
\text { magnification }\end{array}$ & $\begin{array}{l}\text { Resolution } \\
\text { (MPP) }\end{array}$ & $\begin{array}{c}\text { Pixel } \\
\text { dimension }\end{array}$ \\
\hline \multicolumn{4}{|l|}{ Parotid gland } \\
\hline Cross section of parotid gland I & $20 \times$ & 0.497 & $34,176 \times 17,838$ \\
\hline Cross section of parotid gland II & $40 \times$ & 0.247 & $72,000 \times 35,946$ \\
\hline Cross section of parotid gland III & $40 \times$ & 0.247 & $75,600 \times 29,470$ \\
\hline Cross section of parotid gland IV & $40 \times$ & 0.247 & $63,000 \times 27,881$ \\
\hline Cross section of parotid gland V & $40 \times$ & 0.247 & $54,000 \times 37,850$ \\
\hline \multicolumn{4}{|l|}{ Periodontal ligament } \\
\hline Cross section of root, periodontal ligament, alveolar bone I & $40 \times$ & 0.247 & $54,000 \times 49,764$ \\
\hline Cross section of root, periodontal ligament, alveolar bone II & $40 \times$ & 0.247 & $52,199 \times 33,395$ \\
\hline Cross section of root, periodontal ligament, alveolar bone III & $40 \times$ & 0.247 & $50,399 \times 39,356$ \\
\hline \multicolumn{4}{|l|}{ Sublingual gland } \\
\hline Cross section of sublingual gland I & $40 \times$ & 0.247 & $59,399 \times 50,399$ \\
\hline Cross section of sublingual gland II & $40 \times$ & 0.247 & $52,199 \times 50,928$ \\
\hline Cross section of sublingual gland III & $40 \times$ & 0.247 & $52,199 \times 51,262$ \\
\hline \multicolumn{4}{|l|}{ Submandibular gland } \\
\hline Cross section of submandibular gland I & $40 \times$ & 0.247 & $46,800 \times 40,366$ \\
\hline Cross section of submandibular gland II & $40 \times$ & 0.247 & $50,399 \times 52,758$ \\
\hline Cross section of submandibular gland III & $40 \times$ & 0.247 & $55,800 \times 46,282$ \\
\hline \multicolumn{4}{|l|}{ Temporomandibular joint (TMJ) } \\
\hline Cross section of adult TMJ I & $20 \times$ & 0.497 & $39,573 \times 38,056$ \\
\hline Cross section of adult TMJ II & $20 \times$ & 0.497 & $41,372 \times 40,021$ \\
\hline Cross section of adult TMJ III & $20 \times$ & 0.497 & $46,768 \times 37,196$ \\
\hline Cross section of adult TMJ IV & $20 \times$ & 0.497 & $44,969 \times 39,758$ \\
\hline \multicolumn{4}{|l|}{ Tongue } \\
\hline Cross section of tongue I & $20 \times$ & 0.497 & $23,384 \times 28,673$ \\
\hline Cross section of tongue II & $20 \times$ & 0.497 & $23,384 \times 29,572$ \\
\hline Cross section of tongue III & $20 \times$ & 0.497 & $26,981 \times 25,530$ \\
\hline Cross section of tongue IV & $20 x$ & 0.497 & $25,183 \times 21,910$ \\
\hline Cross section of tongue $\mathrm{V}$ & $20 \times$ & 0.497 & $23,384 \times 28,255$ \\
\hline Cross section of tongue VI & $40 \times$ & 0.247 & $48,600 \times 57,230$ \\
\hline Cross section of tongue VII & $20 x$ & 0.497 & $35,975 \times 18,656$ \\
\hline Cross section of tongue VIII & $20 \times$ & 0.497 & $34,167 \times 16,192$ \\
\hline Cross section of tongue IX & $20 \times$ & 0.497 & $34,167 \times 22,859$ \\
\hline
\end{tabular}

and resolution, organized in groups, annotated, and archived on the Histoscope website under the Slide Gallery tab (Figure 1). Currently, the website contains 76 digital slides representing teeth and surrounding oral structures.

\section{Self-Assessment Question Preparation}

We prepared a series of questions (Figure 1D) which were incorporated into the website under the Self-Assessment tab. The questions are grouped to correspond to the Slide Gallery organization, so students can choose to test their knowledge of a specific area of the oral cavity. Currently, we have 35 image-labeling and structure-identification questions on our website, which are randomized each time a user takes the test. 
Table 1. Continued 2

\begin{tabular}{|c|c|c|c|}
\hline \multirow[b]{2}{*}{ Digital slides } & \multicolumn{3}{|c|}{ Properties } \\
\hline & $\begin{array}{c}\text { Scanning } \\
\text { magnification }\end{array}$ & $\begin{array}{c}\text { Resolution } \\
\text { (MPP) }\end{array}$ & $\begin{array}{c}\text { Pixel } \\
\text { dimension }\end{array}$ \\
\hline \multicolumn{4}{|l|}{ Tooth development } \\
\hline Developing tooth germ I & $20 \times$ & 0.497 & $19,786 \times 28,606$ \\
\hline Developing tooth germ II & $20 \times$ & 0.497 & $21,585 \times 30,655$ \\
\hline Bell stage of tooth development I & $20 \times$ & 0.497 & $21,585 \times 42,668$ \\
\hline Bell stage of tooth development II & $20 \times$ & 0.497 & $43,170 \times 24,872$ \\
\hline Bell stage of tooth development III & $20 \times$ & 0.497 & $23,384 \times 39,290$ \\
\hline Bell stage of tooth development IV & $20 \times$ & 0.497 & $26,981 \times 44,779$ \\
\hline Bell stage of tooth development $\mathrm{V}$ & $20 x$ & 0.497 & $23,384 \times 41,182$ \\
\hline Bell stage of tooth development VI & $20 \times$ & 0.497 & $19,786 \times 29,511$ \\
\hline \multicolumn{4}{|l|}{ Tooth eruption } \\
\hline Cross section of erupting tooth I & $20 \times$ & 0.497 & $26,981 \times 27,831$ \\
\hline Cross section of erupting tooth II & $20 \times$ & 0.497 & $30,579 \times 26,829$ \\
\hline Cross section of erupting tooth III & $20 \times$ & 0.497 & $30,579 \times 24,025$ \\
\hline Cross section of erupting tooth IV & $20 \times$ & 0.497 & $30,579 \times 26,253$ \\
\hline Cross section of erupting tooth $\mathrm{V}$ & $20 \times$ & 0.497 & $41,372 \times 27,757$ \\
\hline Cross section of erupting tooth VI & $20 \times$ & 0.497 & $41,372 \times 27,758$ \\
\hline Cross section of erupting tooth VII & $20 \times$ & 0.497 & $39,573 \times 29,828$ \\
\hline Cross section of erupting tooth VIII & $20 \times$ & 0.497 & $39,573 \times 29,826$ \\
\hline Cross section of erupting tooth IX & $20 \times$ & 0.497 & $37,774 \times 21,687$ \\
\hline Cross section of erupting tooth $\mathrm{X}$ & $20 \times$ & 0.497 & $35,975 \times 22,846$ \\
\hline Cross section of erupting tooth XI & $20 \times$ & 0.497 & $39,573 \times 34,683$ \\
\hline Cross section of erupting tooth XII & $20 \times$ & 0.497 & $41,372 \times 32,695$ \\
\hline
\end{tabular}

\section{Design and Layout of the Website}

We aimed for a simple, responsive design with a minimum number of tabs and minimal text for easy navigation. For structural consistency, the title "Histoscope" and the background frame are kept visible on all pages (Figure 1A, 1B). The colors of the headings, tabs, and background were chosen for aesthetic harmony. The contents of the website are organized under six tabs. Digital slides are arranged in fifteen groups under the Slide Gallery tab (Figure 1A, 1B, Table 1 ). The Self-Assessment tab guides users to a series of questions to test their oral histology knowledge. The website also has an option for users to contact the authors and provide feedback for further improvement (Figure 1A).

\section{Incorporation of Interactive Components}

We designed Histoscope as an interactive microscopy tool. This interface's primary goal is to mimic the experience of viewing slides through a real light microscope with the abil- ity to manipulate and view various areas of the histological section, which is rare in the existing online histology databases. Our website was programmed with the Python programming language and uses Flask as a web framework, resulting in a dynamic platform capable of processing user requests and interacting with them accordingly. Users can load a digital slide of their choice in high resolution, rotate the slides as needed, toggle to full screen, and change the magnification in any mouse-pointed location. The digital slides on our website were scanned using the z-stacking technique to provide continuous $\mathrm{z}$-axis information. Zstacking allows for a greater depth of field and a unique ability to magnify a digital image at any given area and maintain a perfectly focused, high-resolution field-of-view (Figure 2). The slide viewer of the website provides an inset telescope box on the upper-right corner of the screen. When zooming in on fields of view, the inset shows a box outlined in red, delineating the exact position of the area being viewed 


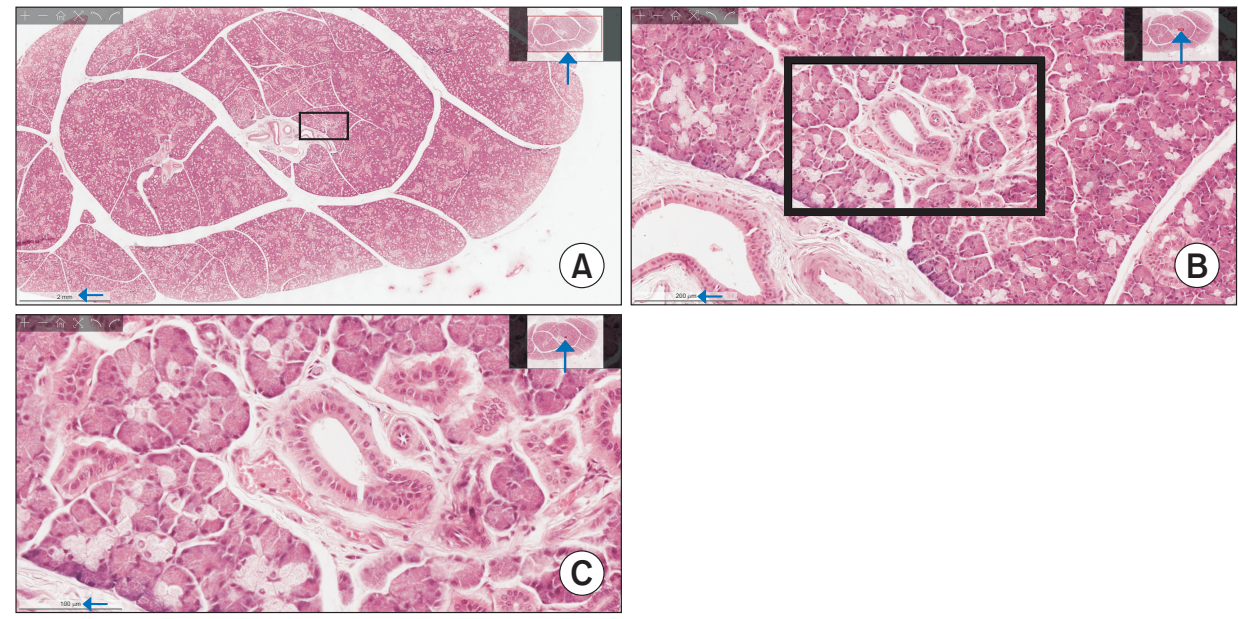

Figure 2. Application of z-stacking to provide in-focus images in high magnification. The slide collection of Histoscope was scanned using the z-stacking technique to allow the ability to magnify the digital slides and still get an entirely focused, high-resolution field-of-view. (A-C) A tissue section of a salivary gland, gradually magnified from low $(A)$ to higher $(B, C)$ magnification. The red box in the inset of each slide indicates the viewing area (arrow) and the scale bar at the bottom left shows the degree of magnification of the slide (arrow). The black box in the middle indicates the area that was being magnified. As the slides are viewed under higher magnification, the scale bar changes (from $2 \mathrm{~mm}$ to $100 \mu \mathrm{m}$ ), and the red box in the inset becomes smaller. Z-stacking enables the user to observe structural details under a higher magnification, mimicking the experience of using a light microscope.

on-screen with respect to the entire viewable field (Figure 2). After magnification, the user can navigate the magnified slide by grabbing the slide with a hand tool or by simply moving the red box in the inset (Figure 3). The slides are annotated by content experts to identify major structural components within a tissue section. Users can either "show" or "hide" the annotations, which allows them to self-test their learning (Figures 1C, 3).

\section{Results}

The completed website includes 76 high-quality and rare sections of the tooth, oral structures, and development of tooth and facial regions, which are not readily available in any public online databases. A complete list of all the digital slides incorporated into our slide gallery is shown in Table 1 . The digital images were scanned using a focus stacking technology to mimic the experience of focusing through a real light microscope (Figure 2). We have included an option to show or hide annotations to enable users to perform active memory recall (Figure 3). Self-assessment questions on our website allow learners to self-test their knowledge on oral histology (Figure 1).

\section{Discussion}

Computer-based technologies to teach histology have in-

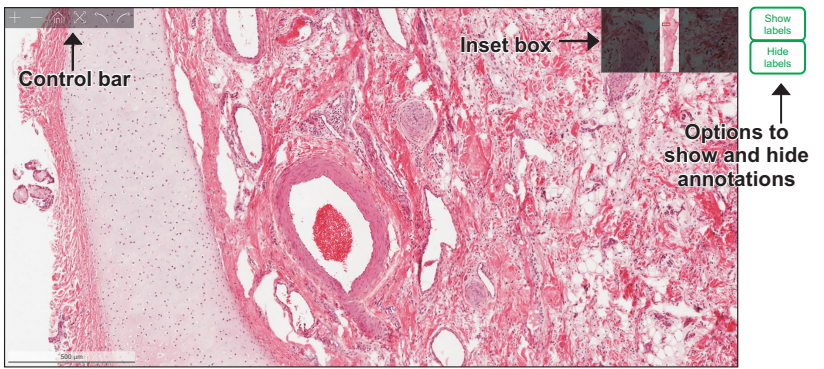

Figure 3. Snapshot from the slide viewer of Histoscope. The user can activate the inset box on the upper-right corner of the slide viewer simply by the mouse pointer's motion. While zooming in, the inset shows a box outlined in red, delineating the exact position of the area being viewed on-screen with respect to the entire viewable field. The control bar in the upper-left corner allows the user the zoom in, zoom out, rotate, and toggle to full-screen mood. "Show Labels" and "Hide Labels" allow users to show or hide the slide annotations.

creased over the years $[3,12,13]$. Virtual microscopy has improved learners' performance in many training programs $[3,5,14]$. Some known limitations of this technology are low graphics resolution and lack of $\mathrm{z}$-axis information $[9,10]$. With Histoscope, we aimed to overcome the shortcomings of previous virtual histology databases and to create an interactive and dynamic platform to mimic the experience of using an actual light microscope. The incorporation of our webbased microscope will stimulate active learning and student 
engagement. Educational theories suggest that adults learn proficiently when they are fully engaged in learning [15]. Active engagement also helps develop critical thinking skills in adults [16]. The use of web-based microscopy in conjunction with an actual light microscope is supported by Mayer's cognitive theory of multimedia learning, which states that using two or more of each of the modalities of delivery, presentation, and sensory systems will support effective learning [17]. We believe that Histoscope will complement traditional teaching with the light microscope and allow students to study histology at their own pace.

\section{Conflict of Interest}

No potential conflict of interest relevant to this article was reported.

\section{Acknowledgments}

The authors sincerely thank Rehabilitation Robotics Lab, University of Alberta for developing our website, Histoscope.

\section{ORCID}

Nazlee Sharmin (https://orcid.org/0000-0002-2408-2333)

Ava K. Chow (https://orcid.org/0000-0002-1972-0310)

Alice S. Dong (https://orcid.org/0000-0002-2620-033X)

Nadine C. Milos (https://orcid.org/0000-0001-5006-3411)

\section{References}

1. Garcia M, Victory N, Navarro-Sempere A, Segovia Y. Students' views on difficulties in learning histology. Anat Sci Educ 2019;12(5):541-9.

2. Capela e Silva F, Rato LM, Lopes OS. Learning and teaching histology: traditional and computational methods. Proceedings of International Conference "Learning and Teaching in Higher Education"; 2010 Apr 15-16; Evora, Portugal.

3. Blake CA, Lavoie HA, Millette CF. Teaching medical histology at the University of South Carolina School of Medicine: transition to virtual slides and virtual microscopes. Anat Rec B New Anat 2003;275(1):196-206.

4. Pantanowitz L, Szymas J, Yagi Y, Wilbur D. Whole slide imaging for educational purposes. J Pathol Inform 2012; 3:46.
5. Kumar RK, Velan GM, Korell SO, Kandara M, Dee FR, Wakefield D. Virtual microscopy for learning and assessment in pathology. J Pathol 2004;204(5):613-8.

6. Helle L, Nivala M, Kronqvist P. More technology, better learning resources, better learning? Lessons from adopting virtual microscopy in undergraduate medical education. Anat Sci Educ 2013;6(2):73-80.

7. Hortsch M. The SecondLook [Internet]. Ann Arbor (MI): University of Michigan; c2019 [cited at 2021 Apr 5]. Available from: https://secondlook.med.umich.edu/ histology.

8. MacPherson BR, Tieman JG. Oral histology: a digital laboratory and atlas [Internet]. Lexington (KY): University of Kentucky; n.d. [cited at 2021 Apr 5]. Available from: http://www.uky.edu/ brmacp/oralhist/index.htm.

9. Rinaldi VD, Lorr NA, Williams K. Evaluating a technology supported interactive response system during the laboratory section of a histology course. Anat Sci Educ 2017;10(4):328-38.

10. Van Es SL, Pryor WM, Belinson Z, Salisbury EL, Velan GM. Cytopathology whole slide images and virtual microscopy adaptive tutorials: a software pilot. J Pathol Inform 2015;6:54.

11. Ray SF. Applied photographic optics: lenses and optical systems for photography, film, video, electronic imaging and digital imaging. 3rd ed. Oxford, UK: Focal Press; 2002.

12. Bloodgood RA, Ogilvie RW. Trends in histology laboratory teaching in United States medical schools. Anat Rec B New Anat 2006;289(5):169-75.

13. Cotter JR. Laboratory instruction in histology at the University at Buffalo: recent replacement of microscope exercises with computer applications. Anat Rec 2001; 265(5):212-21.

14. McBride JM, Drake RL. National survey on anatomical sciences in medical education. Anat Sci Educ 2018; 11(1):7-14.

15. Vella J. On teaching and learning: putting the principles and practices of dialogue education into action. San Francisco (CA): Jossey-Bass; 2008.

16. Karge BD, Phillips KM, Jessee T, McCabe M. Effective strategies for engaging adult learners. J Coll Teach Learn 2011;8(12):53-6.

17. Mayer RE. Multimedia learning. 2nd ed. Cambridge, UK: Cambridge University Press; 2009. 